\title{
Feasibility Investigation of Superelastic Effect Devices for Seismic Isolation Applications
}

\author{
G. Attanasi, F. Auricchio, and G.L. Fenves
}

(Submitted October 2, 2008; in revised form December 10, 2008)

\begin{abstract}
The objective of this work is to investigate the feasibility of a new seismic isolation device concept, in which the restoring force is given by the superelastic effect of shape memory alloys. Seismic isolation is an option for passive protection of structure when an earthquake occurs, because it modifies the structural global response and improves performance. Dynamic responses of proposed innovative SMA isolation system and of traditional bearing device are compared through dynamic time history analyses. Results show that the SMA system is effective in reducing force and displacement demands, dissipating the input seismic energy and limiting the residual displacements.
\end{abstract}

Keywords seismic isolation, shape memory alloys, superelastic effect

\section{Introduction to Seismic Isolation}

When seismic isolation is mentioned, we refer to a technology which mitigates the earthquake effects on buildings and on their vulnerable contents.

\subsection{Base Concept of Seismic Isolation}

The base concept in seismic isolation is to protect the structure from the damaging effects of an earthquake by introducing a support isolating the building from the shaking ground. In the literal sense, the structure (a building, a bridge, or a piece of equipment) is separated from its foundations. After all, in an earthquake, the ground moves and this ground movement causes most of the damages to the structure. So, the principle is simple: to separate the structure from the ground so that the ground will move but the building will not. In practice, a full separation of the structure from its foundations is impossible, and large relative horizontal displacements have to be avoided either during the earthquakes or when other horizontal loads, such as wind, are present. Hence, the common solution is to use a layer, usually between foundation and superstructure, which is more flexible than the other structural elements and is able to transmit the vertical load undergoing lateral displacements without critical damage.

This article is an invited paper selected from presentations at Shape Memory and Superelastic Technologies 2008, held September 21-25, 2008 , in Stresa, Italy, and has been expanded from the original presentation.

G. Attanasi, European School for Advanced Studies in Reduction of Seismic Risk, ROSE School, IUSS Pavia, Pavia, Italy; F. Auricchio, Department of Structural Mechanics, Università degli Studi di Pavia, Pavia, Italy; and G.L. Fenves, Department of Civil and Environmental Engineering, University of California, Berkeley, CA. Contact e-mails: gattanasi@roseschool.it, auricchio@unipv.it, fenves@berkeley.edu.

\subsection{Effects of Seismic Isolation}

Advantages in seismic isolation are evident. First of all, the level of damage is more safely controlled and confined to generally well-replaceable spots; second, an isolation system is usually a nonlinear system which damps and reduces the action demand of the global structure and also limits the transmittable force to the superstructure. However, design of isolated structures has some particular concerns. Practical isolation systems must balance between the extent of force limitation and the acceptable relative displacements across the isolation system during earthquakes. For this purpose, additional damping sources can be useful to reduce both the forces transmitted and the deformations within the structure.

\subsection{Evaluation of Seismic Isolation Effectiveness}

An important dynamic property of every structure is the period of the first mode of vibration (fundamental period). If other parameters are constant, this is a measure of the structural global stiffness. For example, a short masonry building is very stiff, and it is characterized by a short period of vibration, while a frame system skyscraper is more flexible and the period of vibration is longer. In seismic design, the structural period computation leads to the estimation of the seismic load on the buildings. The design spectra summarize the dynamic response of an elastic single degree of freedom (SDOF) system subjected to a ground motion. Seismic design demand is provided in terms of acceleration and displacement as a function of the fundamental period, as shown in Fig. 1.

Nonlinear systems are typically less demanding than the elastic spectra values because the inelastic hysteresis provides some energy dissipation. Hence, if the system is characterized by relevant nonlinear inelastic response, in the design procedure then the elastic spectrum is reduced by a coefficient representing the hysteretic damping effect. For example, if an additional damping component acts and the reduction coefficient is larger, then the hysteresis loop is "fatter."

Given that the isolation layer is more flexible than the rest of the structure, the fundamental period of isolated structures is longer than that of nonisolated structures, changing either the displacement or the acceleration demand. As shown in Fig. 1, 

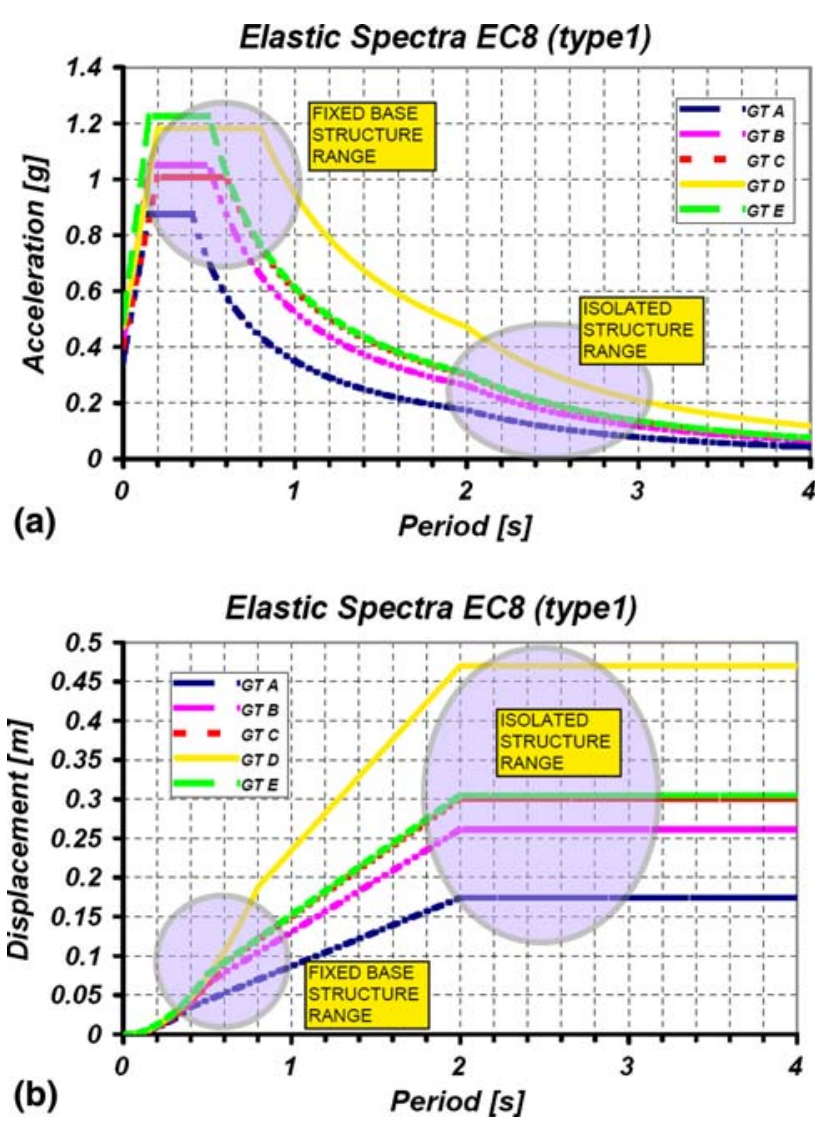

Fig. 1 Elastic design spectra in terms of acceleration (a) and displacement (b) as defined in the Eurocode 8; the different lines refer to different site soil properties; fixed base, and isolated structure domain are shown

usually an isolated structure experiences a smaller acceleration than a fixed base structure and a larger displacement, which in any case is occurring mainly at the isolation level, limiting damage in the superstructure. An extensive and exhaustive description of this topic can be found in Ref 1-4.

\section{Superelastic Isolation Bearing Device Design}

The effectiveness of a hypothetical superelastic isolation device in order to reach the structural design goals is investigated and compared with the traditional isolation device response. In this context, the term superelastic isolation device refers to a bearing system characterized by a nonlinear horizontal force-displacement relation which can be described by a flag-shaped hysteresis.

It is assumed that a bearing system providing a horizontal base shear as a function of displacement such as the one shown in Fig. 2 can be manufactured. At this first stage of work, the device is defined just in terms of hysteresis rule.

The key parameters characterizing the nonlinear behavior of the device in Fig. 2 are:

- $K, \alpha K, r_{1} K, r_{2} K$ are the initial stiffness, the final stiffness, the second stiffness in loading, and the second stiffness in unloading, respectively;

- $V_{\mathrm{d}}, u_{\mathrm{d}}, V_{\mathrm{y}}$, and $u_{\mathrm{y}}$ are the design shear and displacement, the yielding shear and displacement, respectively; $V_{\max }$ is

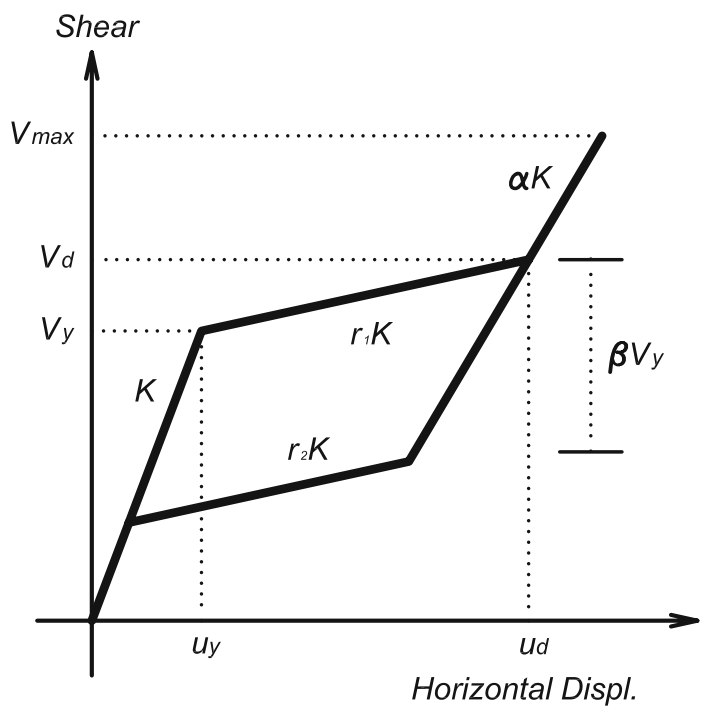

Fig. 2 Parameters for the shear-displacement model of the superelastic isolation device

the maximum lateral force which the device can withstand without breaking; and

- $\beta V_{\mathrm{y}}$ is the shear force difference between the level of force at which the first transformation (when it is loaded) occurs and the level of force at which the second transformation (when it is unloaded) occurs; therefore, $\beta$ is a parameter that measures the dissipation capability of the flag-shaped hysteresis.

The device behaves in the same way in both tension and compression, which implies that the force-displacement relation is symmetric with respect to the origin in the first and third quadrant. The flag-shaped relation is attractive for an isolator device because, in theory, residual displacements are zero at the end of a seismic event. A few of the actual isolation systems avoid the critical failure of the structure, but even if well designed, need to be repaired after the earthquake to bring the system back to the original position with respect to the foundations. On the contrary, an eventual superelastic device is re-centering (Ref 5).

\subsection{Reference Isolation Device}

The superelastic isolation device response is compared with respect to an actual isolation bearing. This is a lead rubber bearing (LRB), which is a bearing composed of a rubber cylinder with a lead core in the middle. The design philosophy of this device is to have the elastic rubber component to transmit the vertical load collaborating for the horizontal stiffness and the lead component that provides a large initial stiffness to resist the nonseismic horizontal load and yields when the design earthquake occurs, providing large hysteretic dissipation.

The device is experimentally characterized (Ref 6). It is produced by $A G O M$ International srl (www.agom.it), and the nominal parameters used for the design purpose are listed in Table 1.

Results of the experimental tests carried out on the bearings are shown in Fig. 3 compared with the elastoplastic hysteresis parameters used in the model. Elastoplastic model is clearly an 


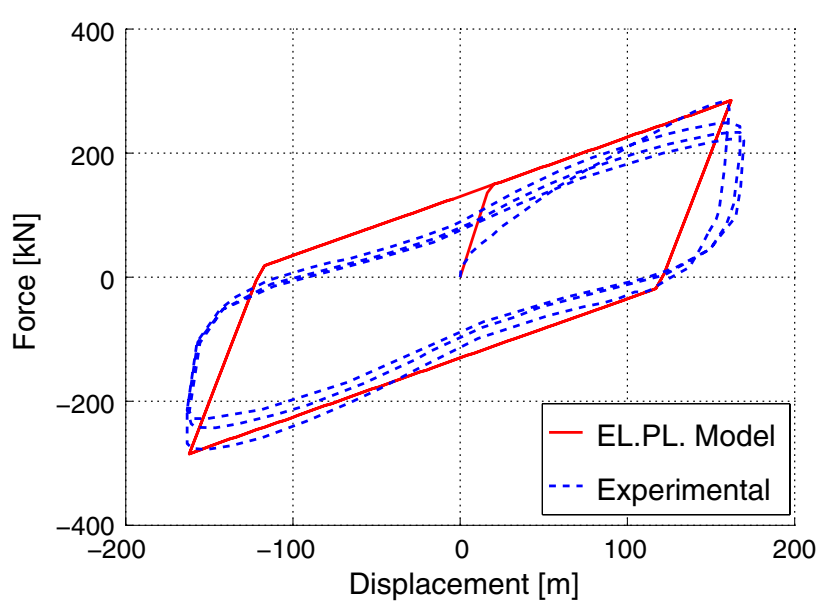

Fig. 3 Force-deformation relations for LRB 500: comparison of experimental test and elastoplastic model (experimental data and model parameters have been provided by $A G O M$ International $\mathrm{srl}$ )

Table 1 Nominal design properties of reference LRB diameter $500 \mathrm{~mm}$ (a) and elastoplastic model parameters (b) [courtesy AGOM International srl] (a) LRB 500

Diameter

Effective horizontal stiffness

Seismic combination vertical load

Seismic design displacement

Hysteretic damping ratio

(b) LRB 500

Yielding shear

Design shear

Yielding displacement

Design displacement

Initial stiffness

Second stiffness
$500 \mathrm{~mm}$

$1.62 \mathrm{kN} / \mathrm{mm}$

$1653 \mathrm{kN}$

$162 \mathrm{~mm}$

$28 \%$

$147 \mathrm{kN}$

$262 \mathrm{kN}$

$17.5 \mathrm{~mm}$

$162 \mathrm{~mm}$

$8.4 \mathrm{kN} / \mathrm{mm}$ $0.8 \mathrm{kN} / \mathrm{mm}$ approximation of the real behavior of the isolator. In particular, the comparison with the experimental results shows that the elastoplastic model does not estimate the initial stiffness or the degradation well. However, for the representation of the general characteristics of the devices, the adopted model is acceptable, since it is exactly the same as the experimental results in terms of secant stiffness at the design displacement, and since it gives a good estimation of the hysteretic energy dissipated and of the residual displacements.

\subsection{Equivalent Superelastic Isolation Device}

The idealized design of an equivalent SMA isolator is based on the properties of the LRB. Starting from the elastoplastic model of the actual device (described in Table 1), an equivalent flag-shaped model related to the hysteresis in Fig. 2 is defined. The SMA device force-displacement relation parameters are summarized in Table 2.

With respect to model in Fig. 2, it is considered that $r_{1}=r_{2}=r$, which means that the device has the same stiffness for loading and unloading in the flag-shaped plateau, and it is assumed that the final hardening occurs far away from the area
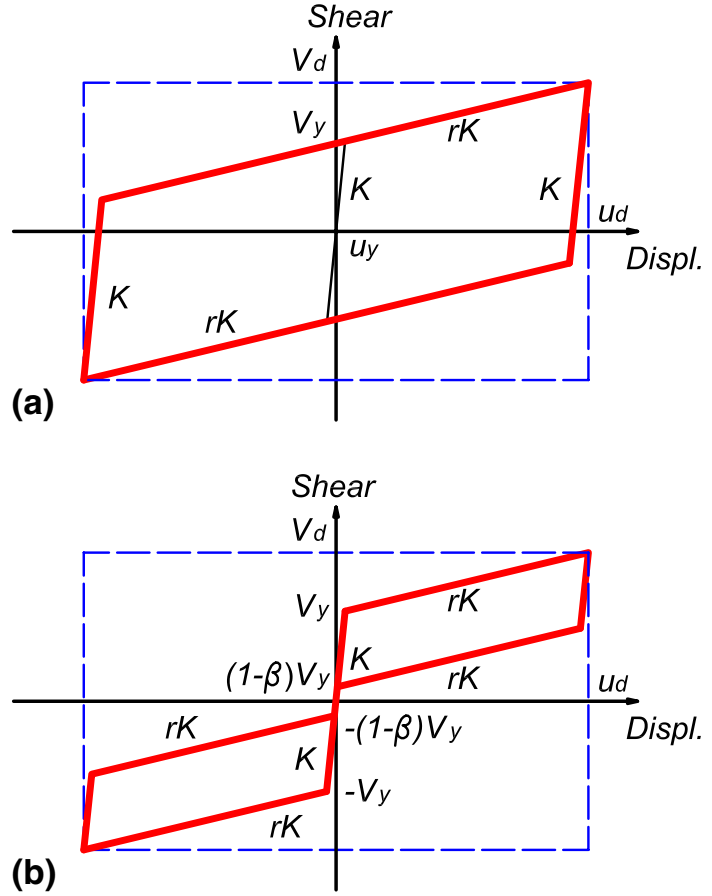

Fig. 4 Hysteretic comparison between LRB elastoplastic model (a) and superelastic flag-shaped model (b)

Table 2 Flag-shaped model parameters for SMA bearing equivalent to LRB diameter $500 \mathrm{~mm}$ (design parameters are the same as those referred to Table 1(b) and as shown in Fig. 2)

\begin{tabular}{lll}
\hline & SMA eq. LRB500 & \\
\hline Yielding shear & $V_{\mathrm{y}}$ & 147 \\
Design shear & $V_{\mathrm{d}}$ & $262 \mathrm{kN}$ \\
Yielding displacement & $u_{\mathrm{y}}$ & $17.5 \mathrm{~mm}$ \\
Design displacement & $u_{\mathrm{d}}$ & $162 \mathrm{~mm}$ \\
Initial stiffness & $k$ & $8.4 \mathrm{kN} / \mathrm{mm}$ \\
Second stiffness & $r k$ & $0.8 \mathrm{kN} / \mathrm{mm}$ \\
\hline
\end{tabular}

of displacement of interest. The dissipation capability of the flag-shaped hysteresis is a parameter for the investigation.

The two systems, the LRB and the SMA, are characterized by the same initial and second stiffness and the same yielding point. The difference between the two systems is only in the hysteresis: the LRB has an elastoplastic force-displacement relation whereas the superelastic device shows a flag-shaped relation, as shown in Fig. 4.

\subsection{Estimation of the Isolation Device Performance}

The seismic displacement and the force demand are investigated by considering the same structural system with two different isolation bearings: one with the isolation layer being given by LRB devices (Fig. 4a), and the other by superelastic devices (Fig. 4b). For the seismic design purpose, the fundamental period is computed through the secant stiffness to the design displacement is used. Provided that $u_{\mathrm{d}}$ is the same 
in both the LRB and in the superelastic models and given that the initial and second stiffness values are also the same, the secant stiffness to the design displacement in our example is the same, and the spectrum in Fig. 1 is achieved with the same period value.

Nevertheless, the hysteresis relation produces important differences in the response evaluation. As mentioned previously, the isolation system is more effective in reducing the seismic input if the hysteresis loop is fatter. The reduction is particularly important especially for the displacement demand, as shown in Fig. 1, where it is critical to accommodate the displacement for long-period structures. Usually in seismic design, the hysteretic damping is computed using the expression (Ref 3):

$$
\xi_{\text {hyst }}=\frac{2}{\pi} \frac{A_{1}}{A_{2}}
$$

in which $A_{1}$ is the area of the hysteresis skeleton curve and $A_{2}$ is the area of the rectangle enveloping the hysteresis relation. Referring to Fig. 4, $A_{1}$ is the area of the portion defined by the continuous line of the force-displacement relation; and $A_{2}$ is the area inside the lined rectangle enveloping the relation. Given the properties of the considered systems, the hysteretic damping given by an elastoplastic force-displacement relation is larger than the one given by a flag-shaped relation. In case of a flag-shaped system with the maximum dissipation capability (meaning $\beta=1$ ), the hysteretic damping in the superelastic device would be exactly one-half with respect the LRB device. If the ductility is defined, which is a measure of the nonlinearity of the system given by the ratio between the yielding displacement $u_{\mathrm{y}}$ and the maximum design displacement $u_{\mathrm{d}}$ as (Ref 4):

$$
\mu=\frac{u_{\mathrm{d}}}{u_{\mathrm{y}}}
$$

The hysteretic damping of the each of two systems is plotted and compared in Fig. 5.

Based on the hysteretic damping, some nonlinear equations have been created to estimate the reduction in terms of the demand both in displacement and in force. They are different as

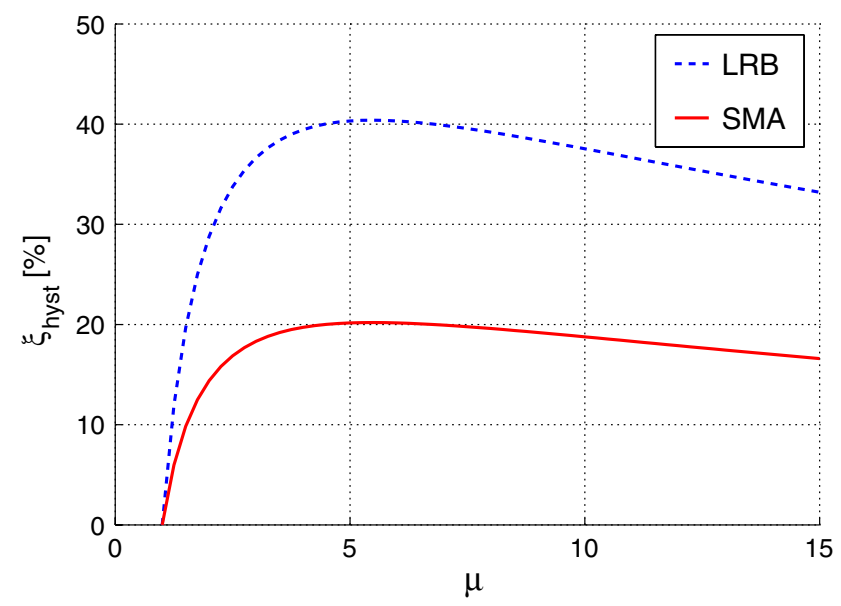

Fig. 5 Hysteretic area-based damping comparison as a function of ductility for superelastic maximum dissipation capability $(\beta=1)$ a function of the formulation, but the expression proposed by Ref 7 is

$$
R_{\xi}=\left(\frac{10}{5+\xi_{\text {hyst }}}\right)^{\alpha}
$$

where $R_{\xi}$ is the reduction factor, and $\alpha$ is 0.5 if the earthquake is a far-field event, and 0.25 if a near-field event is considered (Ref 4).

For design purposes, a reduction factor is used to multiply the spectra shown in Fig. 1 to obtain the relative inelastic spectra (Ref 8). For example, a reduction factor 0.8 means that the system is designed for a force and a displacement equal to the $80 \%$ of the elastic demands given by the spectra, while a reduction factor of 0.4 means that the system is designed only for the $40 \%$ of the elastic demands. The reduction factor is shown as a function of the ductility in Fig. 6, which refers to the hysteretic damping as reported in Fig. 5 and takes into account both the near-fault and the farfield expressions.

The comparison in Fig. 6 shows that according to the hysteretic damping theory, the flag-shaped device is always significantly more demanding than the LRB. Therefore, although in theory the flag-shaped hysteresis isolation system is attractive because it is characterized by zero residual displacements, the results here indicate that the structures have to resist a larger force and are subjected to larger displacement with respect to the actual LRB.

\section{Feasibility Investigation on the Superelastic Isolation Bearing Seismic Behavior}

The equivalent viscous damping approach is a model for seismic design based on the principle that a nonlinear system can be described using a linear system based on the secant stiffness to the design displacement and on an additional viscous damping component (Ref 4 and 8 ). According to this approach, the flag-shaped isolation system is always more

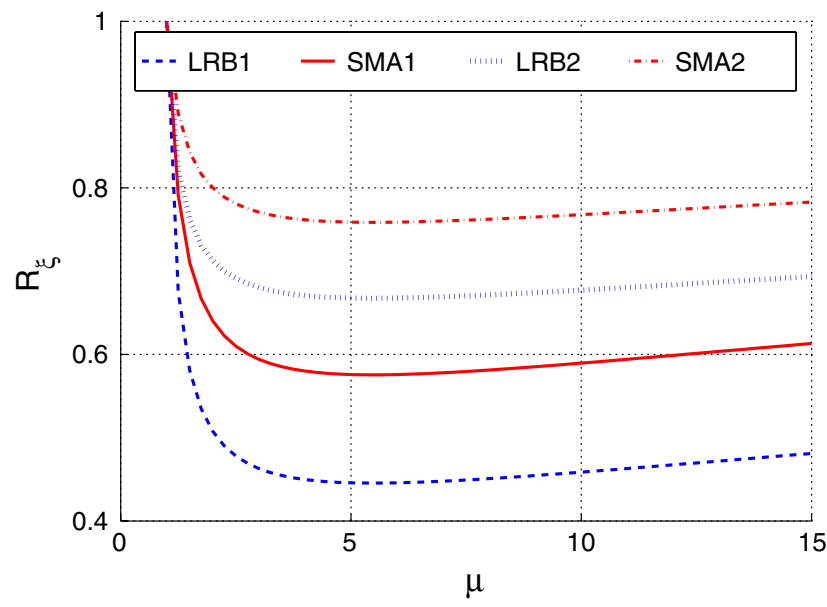

Fig. 6 Force and displacement reduction factors: LRB1 and LRB2 are, respectively, the far-field and the near-field reduction factors computed from LRB hysteretic damping; SMA1 and SMA2 are, respectively, the far-field and the near-field reduction factors computed from SMA hysteretic damping 

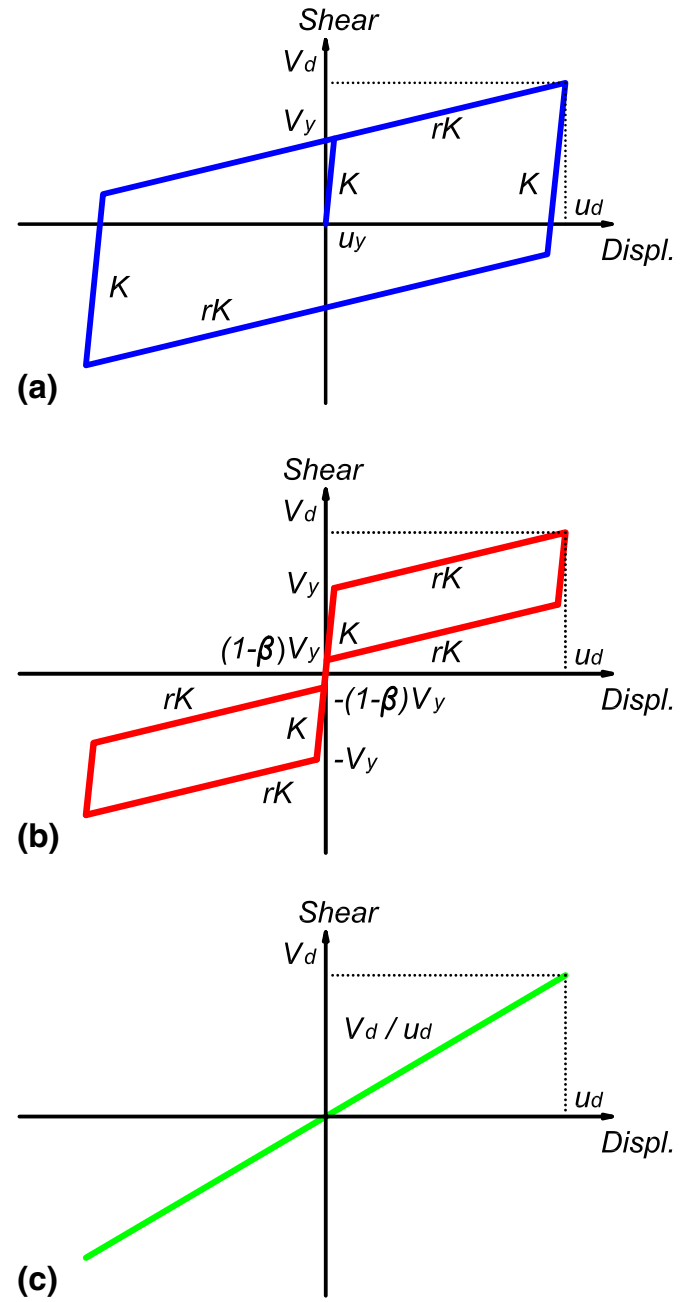

Fig. 7 Base shear-horizontal displacement model for LRB (a), superelastic (b), and equivalent linear elastic with secant stiffness (c) isolation device demanding than an elastoplastic model. In this section, the effective response in terms of the different isolation systems subjected to ground accelerations is investigated, evaluating time history analysis results.

\subsection{Effective Seismic Response Evaluation of Different Hysteresis Isolation Devices}

The investigation is performed considering a SDOF system, in which all the deformation is assumed to occur at the isolation level, with a rigid superstructure affecting the system only as an additional mass. Therefore, the force-displacement relation of the global system is given just by the isolation layer contribution. Fourteen earthquake ground motions are considered and the responses of the following force-displacement relations are compared:

- Elastoplastic model (Fig. 7a). It represents the real LRB device, and the parameters used are those reported in Table 1(b);

- Flag-shaped model (Fig. 7b). It reproduces the shearhorizontal displacement relation of the superelastic device characterized by the design properties reported in Table 2 . The model targets a device that performs similar to the real LRB system in the sense of equivalent shear and displacement capacity and initial and second stiffness;

- Linear elastic model (Fig. 7c). Given the design displacement, $u_{\mathrm{d}}$ and the design shear, $V_{\mathrm{d}}$, which are the same as those in the previous models, the equivalent linear system was developed using parameters reported in Table 1(a).

The elastic damping component is modeled in proportion to the initial stiffness so that its contribution to the three relations is the same provided that the displacement history is also the same (Ref 6). Elastic damping ratio of 5\% in the linear elastic system and of $2.2 \%$ in the elastoplastic and flag-shaped systems are used.
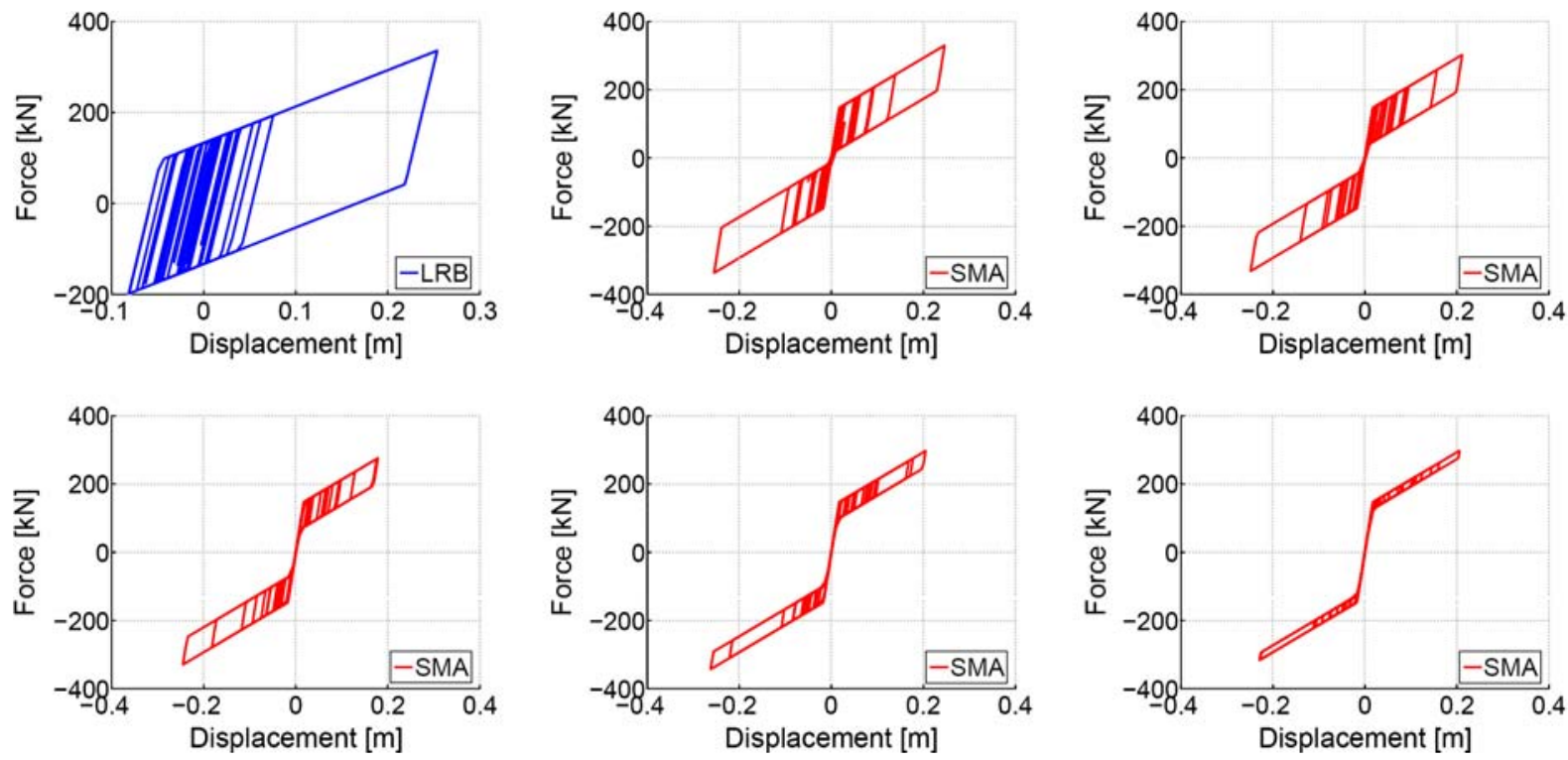

Fig. 8 Elastoplastic and flag-shaped hysteresis considering different dissipation parameters $\beta=0.95 ; 0.75 ; 0.55 ; 0.35 ; 0.15$ used in the analyses (from Tabas (FN) ground motion record analysis) 
Table 3 Ground motion records considered in the analysis

\begin{tabular}{|c|c|c|c|c|c|c|c|c|c|c|}
\hline Earthquake & Date & Location & Comp. & PGA, g & PGV, m/s & PGD, m & Scaling factor & PGA, g & PGV, m/s & PGD, m \\
\hline Tabas & 16 Sep 78 & Tabas stat. & $\mathrm{FN}$ & 0.900 & 1.100 & 0.513 & 0.6257 & 0.563 & 0.689 & 0.321 \\
\hline Tabas & 16 Sep 78 & Tabas stat. & $\mathrm{FP}$ & 0.977 & 1.058 & 0.752 & 0.6382 & 0.624 & 0.675 & 0.480 \\
\hline Erzinican & 13 Mar 92 & Meteor. stat. & $\mathrm{FN}$ & 0.432 & 1.192 & 0.423 & 0.6672 & 0.288 & 0.795 & 0.282 \\
\hline Erzinican & 13 Mar 92 & Meteor. stat. & $\mathrm{FP}$ & 0.457 & 0.581 & 0.295 & 1.2406 & 0.567 & 0.721 & 0.365 \\
\hline Landers & 28 Giu 92 & Lucerne & $\mathrm{FN}$ & 0.713 & 1.360 & 2.298 & 0.6161 & 0.439 & 0.838 & 1.416 \\
\hline Northridge & 17 Jan 94 & Olive View & $\mathrm{FN}$ & 0.732 & 1.222 & 0.310 & 0.5847 & 0.428 & 0.714 & 0.181 \\
\hline Kobe & 16 Jan 95 & Tato & $\mathrm{FP}$ & 0.424 & 0.637 & 0.233 & 0.7109 & 0.302 & 0.453 & 0.165 \\
\hline
\end{tabular}
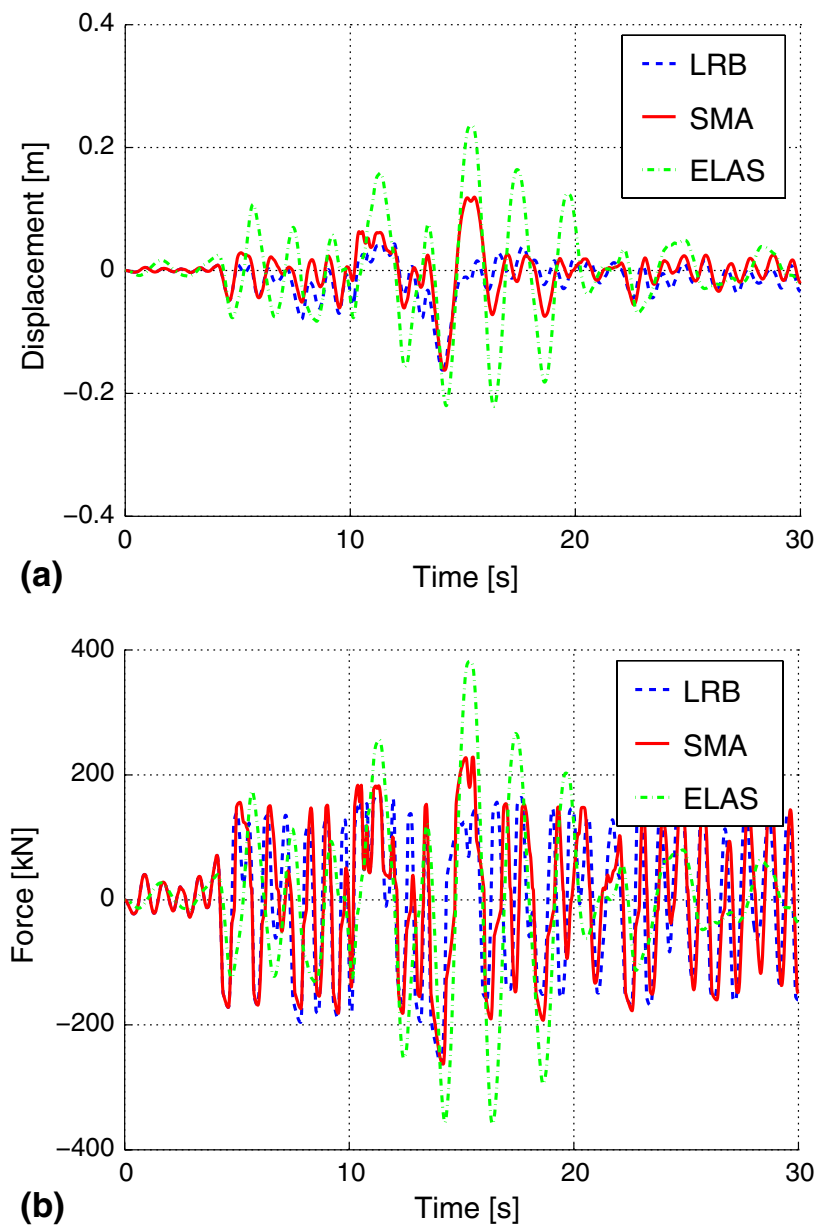

Fig. 9 Comparison between the responses of the three models in terms of displacement (a) and force (b) versus time considering Tabas (FP) record

Even if in theory a high dissipation flag-shaped hysteresis is supposed to be the best for achieving the structural goals, for the sake of investigation, the effect of different dissipation coefficient hysteresis responses is studied. Five cases are studied, with the dissipation parameter (as defined in Fig. 2) spanning between $\beta=0.95$ and 0.15 . All the considered SMA hysteresis models, including the one from elastoplastic models used for comparison, are shown in Fig. 8.

\subsection{Ground Motions for the Time History Analyses}

To include in the analyses the seismic input variability, several ground motions are considered. The base for evaluating

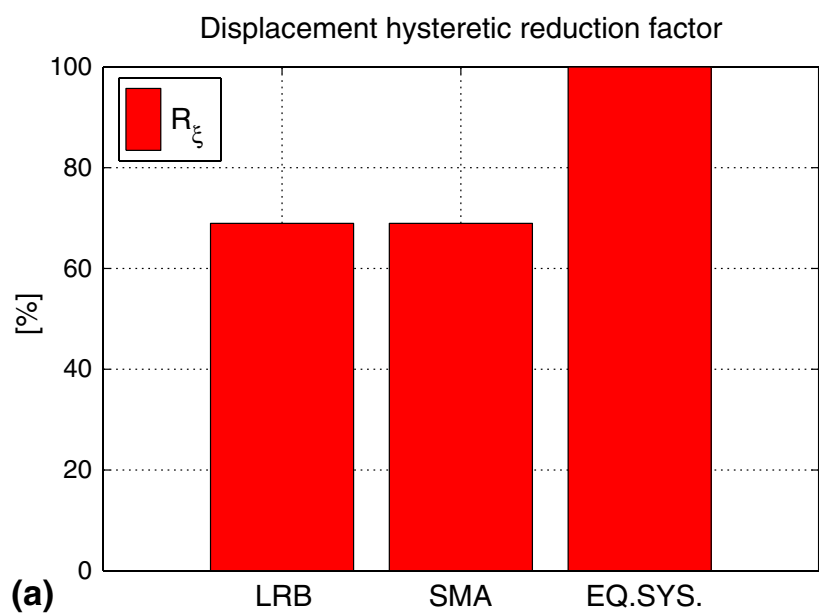

Force hysteretic reduction factor

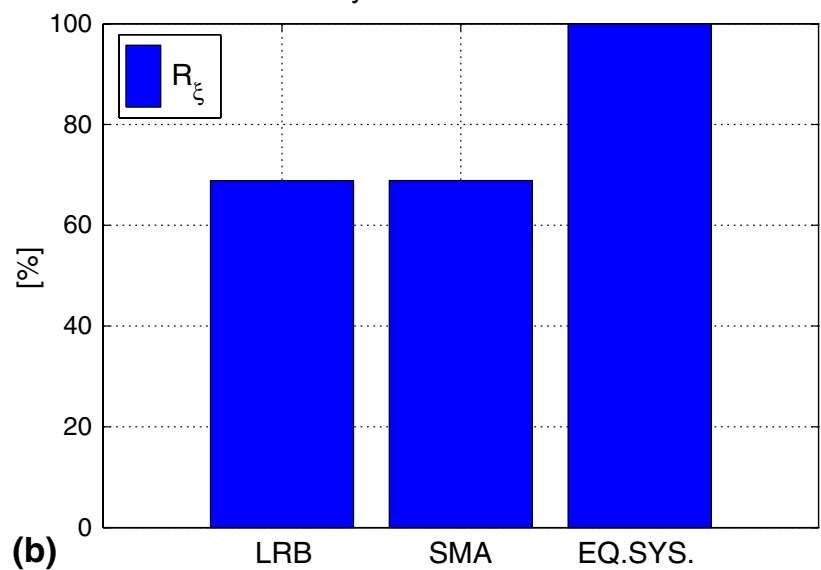

Fig. 10 Maximum displacement (a) and force (b) reduction factor in the three systems considering Tabas (FP) record

the demand is the original design spectra of the actual LRB isolator, which is the Eurocode 8 type $1 \mathrm{PGA}=0.25 \mathrm{~g}$ soil $\mathrm{C}$ (Ref 7), as shown in Fig. 1. Being given the properties of isolated systems, the corner period, which is the period value at which the displacement spectra reach the plateau, has been changed from 2 to $4 \mathrm{~s}$. This induced a more realistic and severe condition in record displacement demand.

The first set of comparison is composed of seven artificial generated ground motions compatible with the design spectra. Seven natural scaled ground motions are also used. The used natural ground motion and the scaling factors are shown in Table 3. 

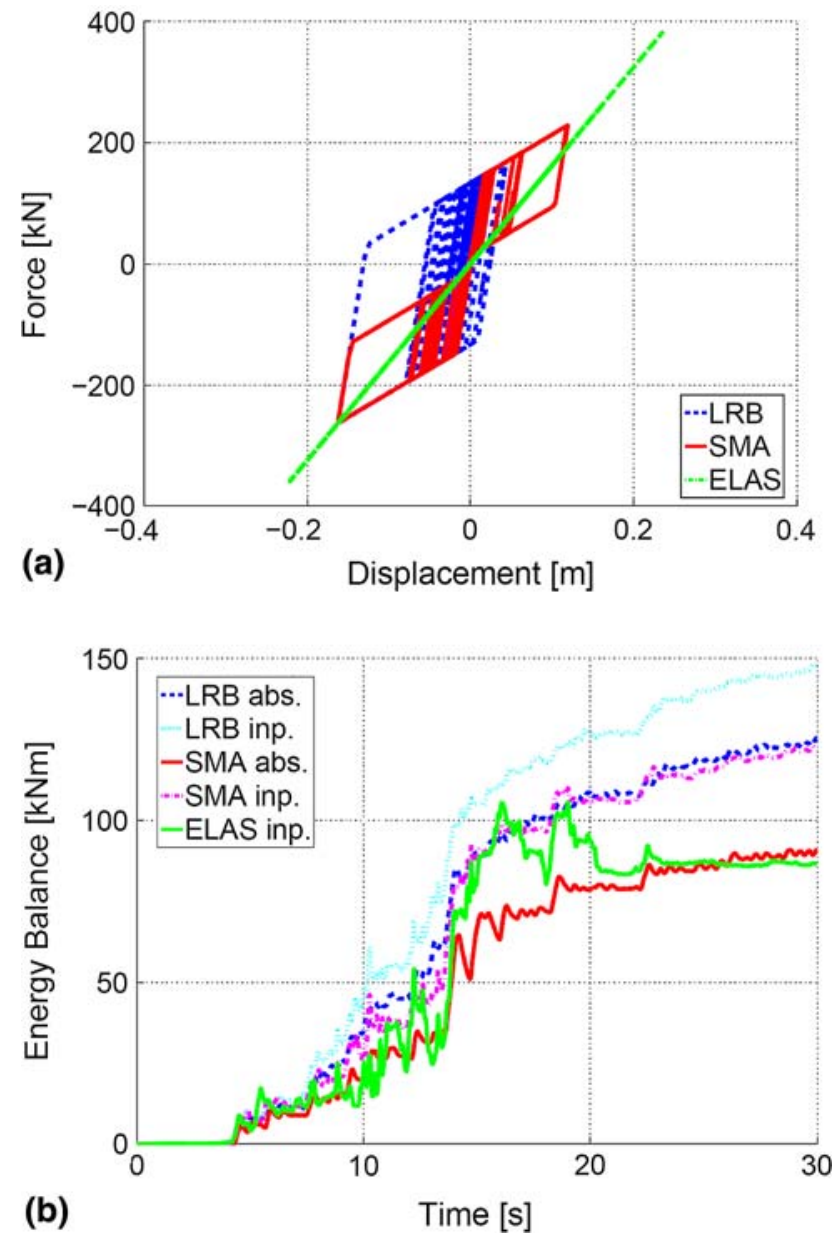

Fig. 11 Comparison of the hysteresis loops (a) and energy balance (b) of the three systems in Tabas (FP) record

\subsection{Time History Analysis Results}

The responses of flag-shaped, elastoplastic, and linear elastic systems are computed considering force and displacement time history demand and system energy balance using Ruaumoko, a nonlinear time history finite element analysis program (Ref 9). Figure 9 shows an example of the system response for a flagshaped model with $\beta$ value equal to 0.95 . The record considered is Tabas (FP). Figure 9(a) shows the history of displacement of the three systems and Fig. 9(b) reports the history of base shear. Using the results shown in Fig. 9, the maximum displacement and shear demand in the linear elastic system with respect to the envelopes in the LRB and SMA systems can be compared. Figure 10 shows the maximum displacement and force demands normalized with respect the maxima in linear elastic system, and Fig. 11 shows the comparison between hysteresis loops, and of energy balance at different times.

Figure 10 shows the exact reduction factor due to hysteresis computed in time histories. The demand of the nonlinear system is smaller than one of the linear systems as expected. What was not anticipated is that the differences between elastoplastic (LRB) and superelastic flag-shaped model (SMA) are very small, even though a significantly vast difference was expected given the differences in the hysteretic area (Fig. 11a).
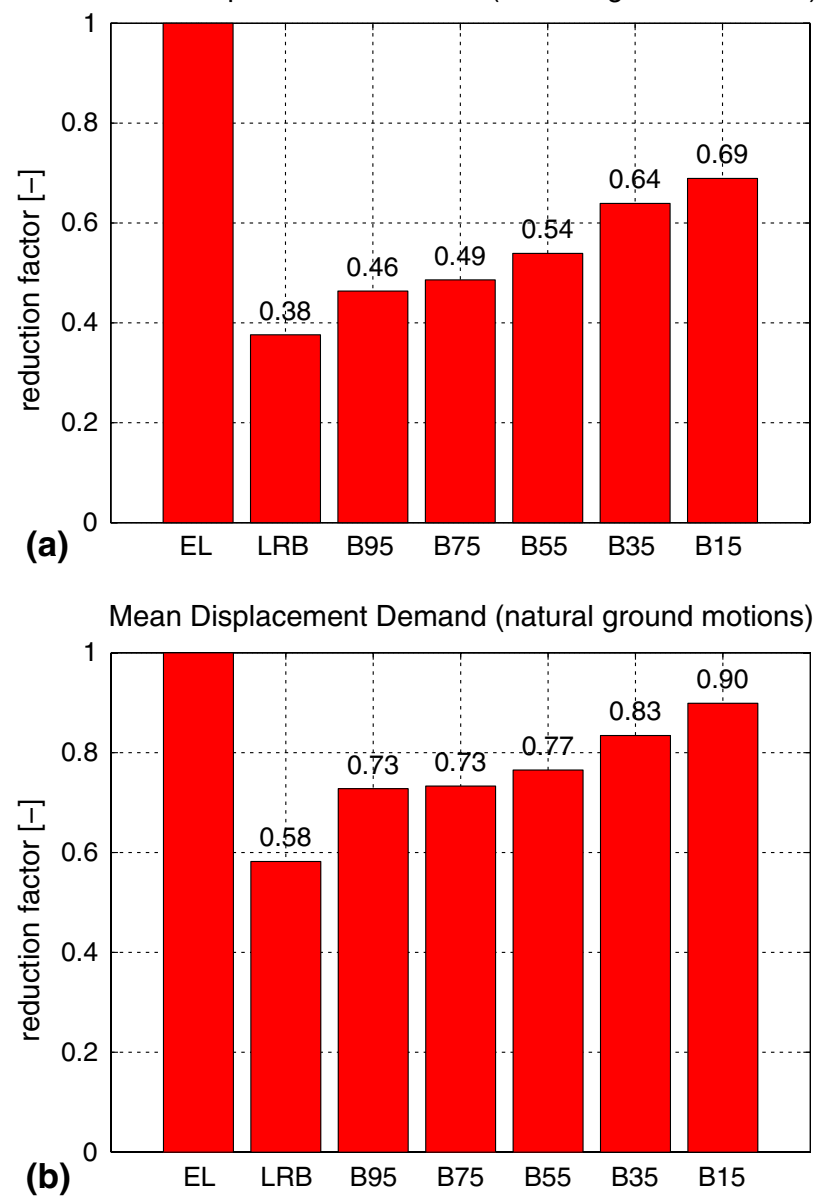

Fig. 12 Displacement mean reduction factor for artificial ground motion (a) and near-fault ground motion (b) considering linear elastic system (EL) and hysteresis in Fig. 8 (LRB, B95, B75, B55, B35, and B15)

The similarity of the results shown in Fig. 10 would be clearer if the energy balance comparison of the systems as shown in Fig. 11(b) is considered. In this set of comparisons, the history of the system input energy in the three models is shown, which is due to the work done by the ground motion on the system. The system responses are different, even if the ground motion is the same for the three different input energy histories (SMA, LRB, and ELAS).

The input energy is converted by the system into components of kinetic, damping, and strain energy. The most interesting component is the strain energy, reported in Fig. 11(b) as absorbed energy, which is integrated with time due to the work done by the reaction force and the lateral displacement of the isolator. The absorbed energy is composed of an elastic and an inelastic contribution, and the comparison focuses on the final inelastic component which is the value at the end of the ground motion, as after some free vibration most of the elastic strain energy is recovered. It is trivial to point out that there is no inelastic strain energy at the end of the history in the linear elastic system. Looking at Fig. 11(b), it turns out that even if the energies in LRB and SMA are different, the ratio of input energies dissipated by inelastic strain given by the hysteresis is very similar. 

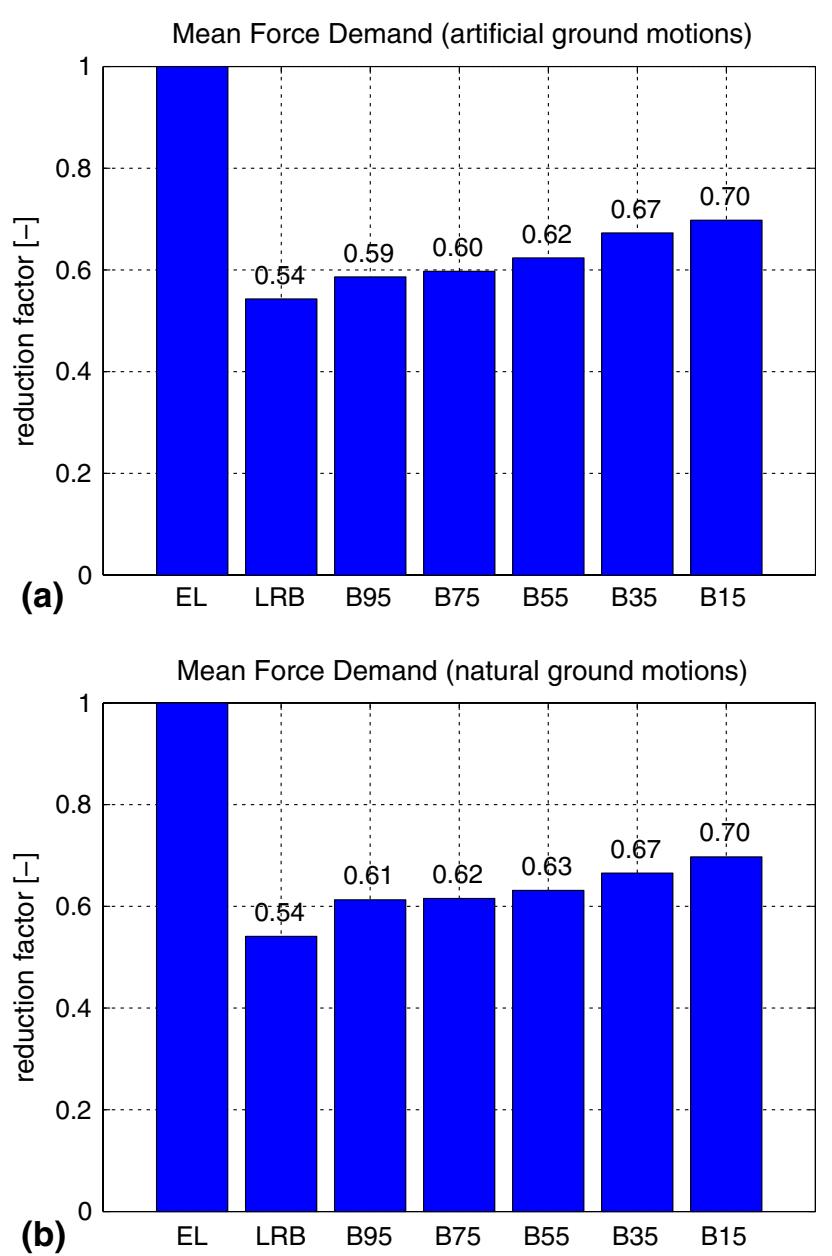

Fig. 13 Mean force reduction factor for artificial ground motion (a) and near-fault ground motion (b) considering linear elastic system (EL) and hysteresis in Fig. 8 (LRB, B95, B75, B55, B35, and B15)

The general comparison of all the ground motion results is shown in Fig. 12 and 13, where mean reduction factors in terms of displacements (Fig. 12) and the forces (Fig. 13) for artificial and natural ground motion records are reported. The comparison is similar to the one shown in Fig. 10, but this time the linear elastic response is compared with the six hystereses found in Fig. 8, which shows the elastoplastic model and the five different dissipation capabilities of the flag-shaped SMA models.

The first and most important result from the comparison is that the differences between the LRB elastoplastic model and superelastic flag-shaped model are small when compared with the linear elastic displacement and force demand for mediumto-high flag-shaped models' dissipation capabilities. Moreover, even by changing the dissipation factor significantly and getting a flag-shaped hysteresis very narrow, the response would not be very different to the most dissipating case. Even though this does not mean the differences are negligible, but these differences turn out to be smaller than the ones expected from those when considering an area-based approach for reduction factor computation.

The response of an idealized SDOF isolation system based on flag-shaped hysteresis considering different dissipations is
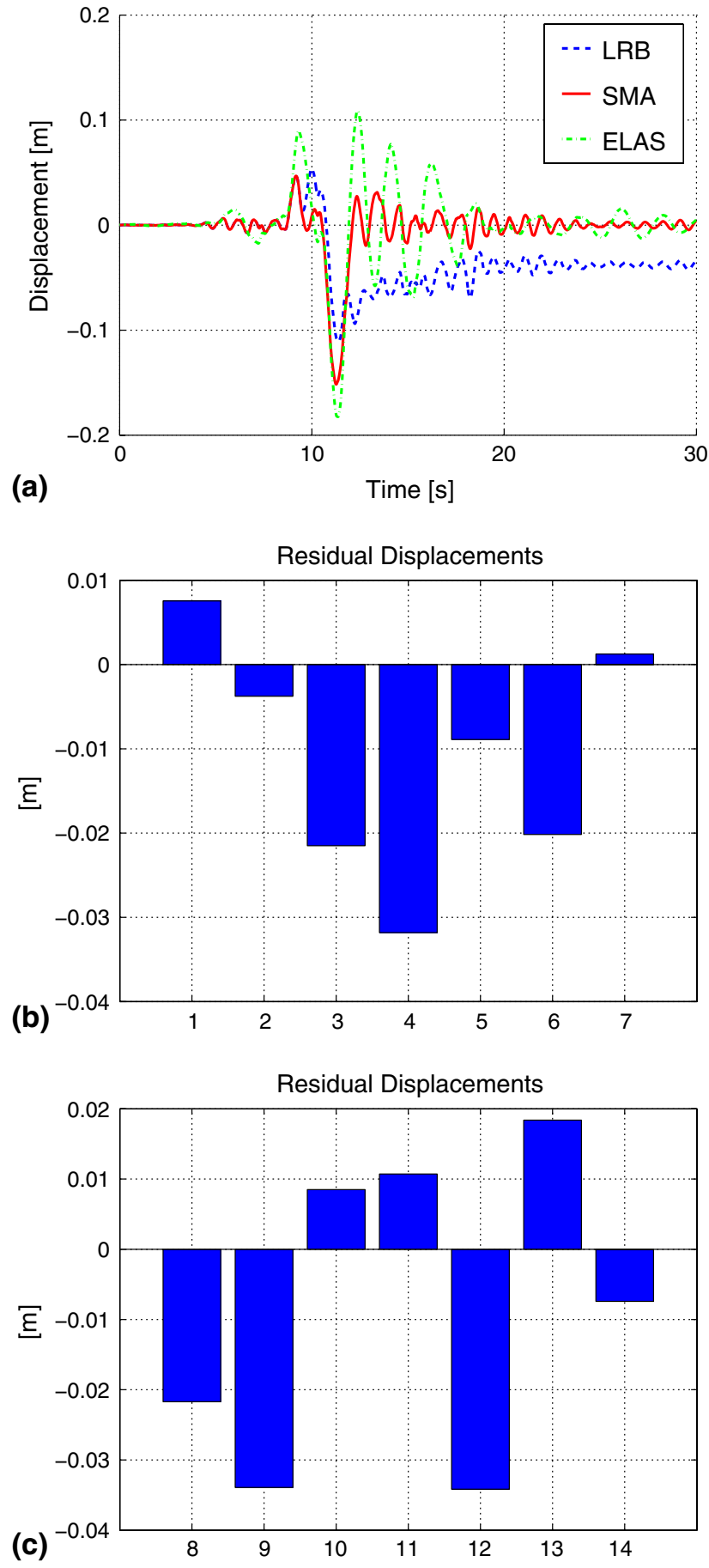

Fig. 14 Displacement-time response from an analysis of the three systems (a) and LRB model residual displacements in artificial (b) and near-fault (c) ground motion

quite good and comparable to the actual lead rubber response at least for a dissipation value in the range $\beta=50 \%$.

Another issue for isolation bearings is the residual displacement. This problem strongly affects the elastoplastic model, especially with a pulse-like near-fault seismic input. Besides, the SMA bearing induces zero residual displacements. An example of displacement-time graph is plotted in Fig. 14, and it shows that the LRB system is characterized by the residuals in all the analyses. 


\section{Conclusions}

The feasibility of superelastic technology application to seismic isolation devices is investigated in this article. According to the area-based hysteretic damping approach, the behavior of a flag-shaped device is expected to be significantly more demanding in terms of base shear and displacement than that of an actual device.

The evaluation of the response data from time history analyses has been considered as the most suitable method to study the problem in detail. The behavior of a model representing a conventional $\mathrm{LRB}$ device, the behavior of a superelastic technology device, and of an equivalent linear elastic model have been compared. Results show that the overall behavior of the isolation system characterized by the flag-shaped hysteresis with a medium-to-high dissipation capability is close to the response of an elastoplastic isolation system. In particular, the displacement and force demands of LRB and SMA normalized with respect to the linear elastic secant model response are quite similar for large SMA dissipation capability. Besides, the differences between the ratios of absorbed energy and input energy are small.

Based on the investigation results, the SMA is possible to be used in seismic isolation and can lead to several advantages. SMA devices have good recentering capability. Moreover, superelastic devices are characterized by energy dissipation and numerical investigations have demonstrated that it is comparable, for its influence on the response, to the dissipation of actual highly dissipating devices.

\section{References}

1. R.I. Skinner, W.H. Robinson, and G.H. McVerry, Seismic Isolation, Wiley, Chichester, UK, 1993

2. F. Naeim and J.M. Kelly, Design of Seismic Isolated Structures. From Theory to Practice, Wiley, New York, 1999

3. C. Christopoulos and A. Filiatrault, Principles of Passive Supplemental Damping and Seismic Isolation, IUSS Press, Pavia, Italy, 2006

4. M.J.N. Priestley, G.M. Calvi, and M.J. Kowalsky, Displacement-Based Seismic Design of Structures, IUSS Press, Pavia, Italy, 2007

5. R. DesRoches and B. Smith, Shape Memory Alloys in Seismic Resistant Design and Retrofit: A Critical Review of Their Potential and Limitations, J. Earthquake Eng., 2003, 7, p 1-15

6. G. Attanasi, "Feasibility Assessment of Innovative Isolation Bearing System with Shape Memory Alloys," Master thesis, ROSE School, University of Pavia, Italy, 2008

7. Eurocode 8. Design of Structures for Earthquake Resistance, Part I: General Rules, Seismic Actions and Rules for Buildings. pre-ENV 1998-1

8. D.N. Grant, C.A. Blandon, and M.J.N. Priestley, Modelling Inelastic Response in Direct Displacement Based Design, IUSS Press, Pavia, Italy, 2005

9. A.J. Carr, Ruaumoko Manual Volume 4: User Guide to Associated Programs, Department of Civil Engineering, University of Canterbury, New Zealand, 2007 